\title{
Análisis de la analgesia en la baja de combate. Experiencia de la Sanidad Militar española
}

\author{
Navarro Suay R. ${ }^{1}$, Castillejo Pérez S. ${ }^{1}$
}

Sanid. mil. 2012; 68 (1): 8-16; ISSN: 1887-8571

\begin{abstract}
RESUMEN
Antecedentes y Objetivos: La analgesia del herido ha jugado un papel trascendental en la medicina militar. En la actualidad, continúa siendo un reto médico, táctico y logístico dentro del tratamiento integral de la baja en combate. El objetivo de este estudio es valorar la homogeneidad de los fármacos analgésicos administrados, del momento de inicio del tratamiento, del número y tipos de vías de acceso para administración de analgesia, así como del tipo de anestesia realizada en las bajas por arma de fuego o por artefacto explosivo atendidas en el ROLE 2E español de Herat (Afganistán) entre 2005 y 2008, siguiendo un índice de gravedad anatómico (NISS) de las lesiones. Material y métodos: Se realiza un estudio observacional, retrospectivo, obteniendo una muestra de 256 pacientes. Resultados: Los fármacos analgésicos más empleados fueron los AINEs (73\%), seguidos de los mórficos mayores (44\%), coadyuvantes (29\%), mórficos menores (21\%) y ketamina (12\%). La analgesia se realizó a nivel prehospitalario en un $61 \%$ y a nivel intrahospitalario en un 31\% de los casos. La vía de administración más frecuente fue la intravenosa (79\%). En el 75\% sólo se consiguió un acceso para medicación analgésica. El procedimiento anestésico más empleado fue la anestesia general (32\%). Conclusiones: El tratamiento analgésico prestado a las bajas en combate de la muestra es homogéneo en cuanto a los fármacos empleados, el tipo y número de vías de administración conseguidas. Sin embargo es heterogéneo en cuanto al tipo de AINE elegido y al inicio en la administración de los fármacos analgésicos.
\end{abstract}

PALABRAS CLAVE: Analgesia, combate, dolor, sanidad militar española, Afganistán

\section{Analgesia in the management of the combat casualty. Experience of the Spanish Medical Service SUMMARY}

Antecedents and Objectives: The analgesia of the wounded has played a vital role in military medicine. Nowadays it still is a medical, tactical and logistical challenge in the integral management of the combat casualty. The objective of this study is to evaluate the homogeneity of the analgesic drugs used, starting point of the treatment, number and type of administration routes, as well as the type of anesthesia used in gunshot or explosive device casualties in the Spanish Role 2 in Herat (Afghanistan) between 2005 and 2008, in accordance with an anatomical injury severity score (NISS) of the injuries. Material and Methods: We carried out an observational, retrospective study with a sample of 256 patients. Results: The drugs most commonly used were the NSAIDs (73\%) followed by major morphine derivatives (44\%), adjuvants (29\%), minor morphine derivatives (21\%), and ketamine (12\%). In $61 \%$ of the cases the analgesia was administered in the prehospital setting and in $31 \%$ in the hospital. The most common administration route was intravenous (79\%). In 75\% of the cases only one administration route was obtained for analgesia. General anesthesia was the most common anesthetic procedure (32\%). Conclusions: Analgesic treatment of the combat casualties studied was homogeneous in terms of drugs used and number and types of administration routes. However it was heterogeneous in reference to the chosen type of NSAIDs and initiation of analgesic drug administration.

KEYWORDS: Analgesia, Combat, Pain, Spanish Medical Service, Afghanistan.

\section{INTRODUCCIÓN}

La analgesia siempre se ha considerado uno de los pilares fundamentales de la asistencia médica a la baja de combate ${ }^{1}$. Esta opinión sigue vigente en los actuales conflictos de Irak y Afganistán.

Tras los atentados del 11 de septiembre del 2001 en Estados Unidos, el Consejo de Ministros autorizó la participación de unidades militares españolas en la Fuerza Internacional de Asistencia

\footnotetext{
Cap. Médico. Escuela Militar de Sanidad.
}

Dirección para correspondencia: Capitán Médico Ricardo Navarro Suay. Grupo de Escuelas de la Defensa. Escuela Militar de Sanidad. Camino de los Ingenieros n. ${ }^{\circ} 6$. CP: 28071. Madrid. E-mail: r navarro suay@yahoo.es

Recibido: 28 de junio de 2011

Aceptado: 15 de diciembre de 2011 para la Seguridad (International Security Assistance Force, ISAF) en apoyo del gobierno interino afgano. España asumió el mando de la Base de Apoyo Avanzado (Foward Support Base, FSB) de Herat (Afganistán)2 . Dentro de la FSB, se encuentra el Hospital Militar español (ROLE-2E) y los equipos de aeroevacuación médica (MEDEVAC). En el ROLE-2E español de Herat, se atiende tanto a personal civil, como a efectivos militares desplegados en la región, que presentan enfermedades o lesiones relacionadas con el combate. Por capacidad, material y personal es considerada por la OTAN la instalación médica de referencia de las cuatro provincias que forman la región oeste de Afganistán³.

Dentro del abordaje multidisciplinar, el dolor puede ser tratado farmacológicamente según la escala analgésica propuesta por la Organización Mundial de la Salud. Esta escalera de tratamiento está compuesta por cuatro escalones: primer escalón (antiinflamatorios no esteroideos -AINES-: paracetamol, metamizol, ibuprofeno, ketorolaco...), segundo escalón (opioides débiles: codeína, trama- 
Tabla 1. Índice New Injury Severity Score (NISS).

\begin{tabular}{|lll|}
\hline Regiones corporales & Gravedad & Probabilidade fallo multiorgánico \\
\hline Cabeza y cuello & 1: Leve & Grado I: NISS $15-24: 4 \%$ \\
Cara & 2: Moderada & Grado II: NISS $>25: 14 \%$ \\
Tórax & 3: Grave sin riesgo vital & Grado III: NISS $\geq 25: 54 \%$ \\
Abdomen-Pelvis & 4: Grave con riesgo vital & Grado IV: NISS $\geq 25$, \\
Extremidades-Pelvis ósea & 5: Crítica & $>6$ unidades de Hematíes y \\
General o Externa & 6: No supervivencia & Láctico $\geq 2.5: 75 \%$ \\
\hline
\end{tabular}

Cálculo del NISS: Asignar a cada lesión un coeficiente dependiendo de la gravedad. Sumar los cuadrados de los tres coeficientes más elevados.

dol...), tercer escalón (opioides potentes extraespinales: cloruro mórfico, fentanilo...) y cuarto escalón (opioides potentes espinales). Con todos ellos, también se pueden administrar fármacos coadyuvantes (anticonvulsionantes, antidepresivos tricíclicos, ansiolíticos, antipsicóticos... $)^{4}$. En la actualidad, existen múltiples vías de administración de este tipo de fármacos ${ }^{5,6}$, destacando la vía oral, intravenosa, intramuscular, intraósea, submucosa o transmucosa, que permiten iniciar y mantener el tratamiento analgésico del paciente ${ }^{7}$.

Con la intención de evaluar, estratificar, prevenir y prepararse para atender el traumatismo, se han descrito algunos sistemas de medida con la finalidad de determinar la gravedad y el pronóstico de estas víctimas. El índice de gravedad NISS (New Injury Severity Score) se emplea frecuentemente en el paciente politraumatizado ${ }^{8}$ (Tabla 1).

Debido a las particularidades tácticas, logísticas, formativas y asistenciales del medio militar, varias Sanidades Militares de nuestro entorno abogan por establecer un protocolo terapéutico según los diferentes escalones. Su intención es normalizar el tratamiento analgésico de la baja de combate y con ello facilitar el apoyo sanitario desde su inicio, simplificar el aprovisionamiento de los recursos sanitarios, favorecer la enseñanza y recomendar una determinada asistencia terapéutica ${ }^{9-11}$.

La hipótesis de este trabajo de investigación fue valorar si el tratamiento analgésico administrado a las bajas en combate de la muestra es homogéneo. El objetivo principal fue determinar si el tratamiento analgésico administrado a las bajas por arma de fuego o por artefacto explosivo atendidas por oficiales médicos españoles desplegados en la región oeste de Afganistán, seguía un patrón homogéneo. Los objetivos secundarios fueron determinar la homogeneidad en el tratamiento, dependiendo del grupo de fármacos analgésicos empleado, del momento de inicio de la administración de analgesia, de la vía de administración elegida, y valorar la creación de un programa de tratamiento del dolor.

\section{MATERIAL Y MÉTODO}

Se realizó un estudio observacional, descriptivo, longitudinal y retrospectivo, entre los años 2005 y 2008. Se eligió la siguiente muestra: todo personal civil y militar, que tras sufrir herida por arma de fuego o por artefacto explosivo en la región oeste de Afganistán, fuese atendido en el ROLE-2E español de Herat (Afganistán) desde diciembre del 2005 a diciembre del 2008. Como muestreo, se selecciona todo el universo de la población a estudio. No se empleó ningún criterio de exclusión. Se consiguió un tamaño muestral de $n=256$.

Las variables incluidas en este estudio fueron de dos tipos: independientes: agente lesional (arma de fuego, explosivo -IED: Im- provised Explosive Device- o arma de fuego más explosivo), área anatómica afectada (cabeza-cuello, tórax, abdomen, miembros superiores, miembros inferiores), empleo de medios de protección (casco, chaleco de protección, blindaje) y gravedad según índice NISS (leve, moderada, grave); y dependientes: mortalidad (fallecimiento, vivo), necesidad de intervención quirúrgica (sí, no), necesidad de ingreso en Unidad de Cuidados Intensivos -UCI- (sí, no), tipo de analgesia administrada por grupos farmacológicos (AINEs -paracetamol, metamizol, ibuprofeno, ketorolaco, otros- mórficos menores -codeína, tramadol-, mórficos mayores -cloruro mórfico, fentanilo-, coadyuvantes y ketamina), vías de administración de fármacos analgésicos (vía oral, vía intravenosa, vía intramuscular, vía intraósea, vía submucosa y vía transmucosa), momento de iniciar el tratamiento analgésico (prehospitalario, intrahospitalario), tipo de anestesia realizada (local, regional, general) y sociodemográficas y de control: sexo, edad, civil/militar, zona geográfica de procedencia, y transporte sanitario (ambulancia, helicóptero). El material para medir dichas variables fue una ficha de recogida de datos e índices anatómicos. El método de medida de las variables consistió en revisar 12.256 historias clínicas de todos los pacientes que fueron atendidos en el ROLE-2E español entre diciembre de 2005 y diciembre de 2008. Este estudio fue aprobado por la máxima autoridad sanitaria de la OTAN en la región oeste de Afganistán.

El método estadístico fue descriptivo. Como índices de la tendencia central y de dispersión de las variables cuantitativas de las distribuciones muestrales se emplearon la media aritmética y la desviación estándar. Para las variables categóricas se emplearon sus frecuencias absolutas y relativas en tantos por ciento (\%). Empleamos el índice de concordancia, que establece que la coincidencia entre observadores se puede considerar de relevancia moderada a partir de un $65 \%$. En todos los casos, como grado de significación estadística se empleó el criterio de $\mathrm{p}<0,05$. La aplicación estadística fue el paquete $\operatorname{SPSS}^{\circledR}$ versión 15.

\section{RESULTADOS}

Durante el periodo del estudio, se recopilaron un total de 256 bajas que habían sufrido lesiones por arma de fuego o artefacto explosivo en la región oeste de Afganistán y que fueron tratados en el ROLE-2E español de Herat durante los años 2005 y 2008 (Tabla 2).

El 37\% (n=32) de las bajas que ingresaron en UCI, el 20,4\% $(n=29)$ de los que fueron intervenidos quirúrgicamente, el $12,1 \%$ $(n=25)$ que estuvo hospitalizado, el 34,3\% ( $n=11)$ de los evacuados a un escalón superior y el 70\% $(\mathrm{n}=7)$ de los fallecidos, estaban valorados como NISS grave. 
Tabla 2. Datos generales de la muestra.

\begin{tabular}{|c|c|}
\hline Sexo & Varón $96 \%(\mathrm{n}=246)$. Mujer 4\% $(\mathrm{n}=10)$ \\
\hline Edad & Entre 25 y 29 años $39 \%(n=101)$ \\
\hline Grupo & Afghan National Army $44 \%(n=112)$ \\
\hline Años & $\begin{array}{l}\text { Año 2006: } 19 \%(n=49) \text {. Año 2007: 36\% ( } n=92) \text {. Año 2008: } \\
44 \%(n=114)\end{array}$ \\
\hline Estación & Verano $36 \%(n=93)$. Otoño $33 \%(n=85)$ \\
\hline Procedencia & Farah - Sur- $53 \%(n=134)$. Badghis - Norte- $21 \%(n=54)$ \\
\hline Evacuación & MEDEVAC - aeroevacuación- 76\% ( $\mathrm{n}=194)$ \\
\hline Agente & IED $71 \%(n=183)$. Arma de fuego $29 \%(n=29 \%)$ \\
\hline Lesión & M. inferiores $48 \%(n=123)$. M. superiores $39 \%(n=99)$ \\
\hline $\mathrm{N}^{\circ}$ lesiones & 1 región afectada $55 \%(\mathrm{n}=142) .2$ regiones afectadas $23 \%(\mathrm{n}=59)$. \\
\hline Protección & Medidas de protección $25 \%(\mathrm{n}=62)$ \\
\hline Mortalidad & $\begin{array}{l}6 \%(n=15) \text {. Prehospitalaria: } 33 \%(n=5) \text {. Intrahospitalaria: } \\
67 \%(n=10)\end{array}$ \\
\hline
\end{tabular}

De las 256 bajas analizadas, 206 (80\%) fueron hospitalizadas en el ROLE-2E español, 142 (55\%) fueron intervenidas quirúrgicamente y 86 (34\%) ingresaron en la unidad de cuidados intensivos, con una estancia media en esta unidad de 2,8 días. Por otro lado, a 63 pacientes $(24,6 \%)$ se les practicaron procesos quirúrgicos menores.

\section{Analgésicos (Figura 1)}

\section{Administración de AINES:}

Del total de la muestra, a 189 pacientes (73\%) se les administró uno o varios AINES. De ellos, a 63 (33\%) se les administró un analgésico AINE, a 112 (59\%) se les administró dos AINES y a 14 (7\%) tres diferentes tipos de AINE. A 99 pacientes (38\%) se les administró paracetamol, a 130 pacientes (50\%) se les administró metamizol, a 28 pacientes $(10 \%)$ se les administró ibuprofeno y a 72 ketorolaco $(28 \%)$

\section{Administración de opioides menores}

De la muestra seleccionada, a 55 pacientes (21\%) se les administró un opioide menor, siendo en todos los casos $(n=56,100 \%)$ tramadol el opioide menor elegido. A ningún miembro de la muestra seleccionada se le administró codeína.

\section{Administración de opioides mayores}

A 114 pacientes (44\%) se les administró algún opioide mayor, siendo el cloruro mórfico elegido en 47 pacientes (41\%) y el fentanilo en $105(59 \%)$.

\section{Administración de coadyuvantes:}

Del conjunto de la muestra, a 75 pacientes $(29 \%)$ se les administró un coadyuvante.

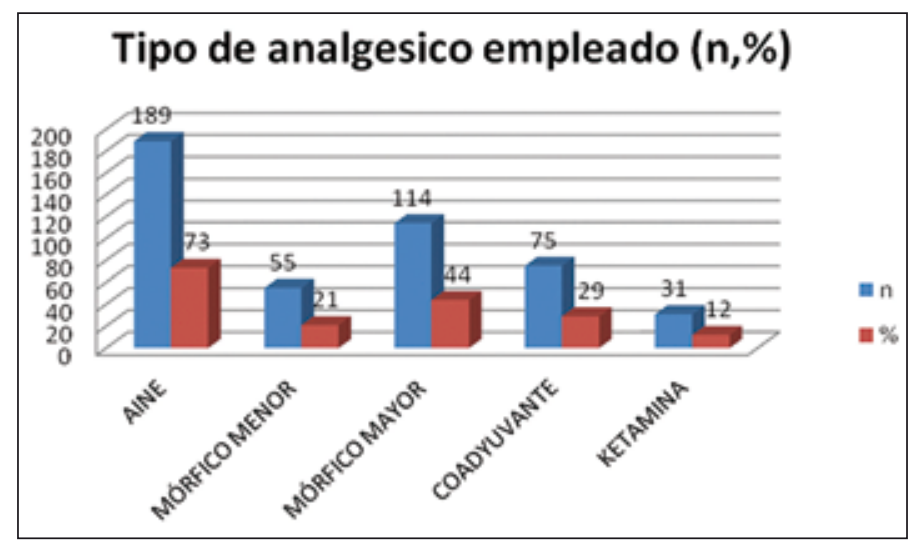

Figura 1. Tipo de fármaco analgésico empleado ( $n$ y \%).

\section{Administración de Ketamina}

La ketamina se administró en 31 pacientes (12\%) de la muestra.

\section{Inicio de la analgesia}

A 158 pacientes (61\%) se les administró analgesia a nivel prehospitalario, mientras que a 98 pacientes $(39 \%)$ se les inicio la pauta analgésica a nivel intrahospitalario (Figura 2). En 158 pacientes (61\%) el tratamiento analgésico a nivel prehospitalario se continuó en el hospital. El 100\% de los pacientes de la muestra seleccionada recibieron analgesia en algún momento de la asistencia (prehospitalario o intrahospitalario).

\section{Inicio del tratamiento analgésico}

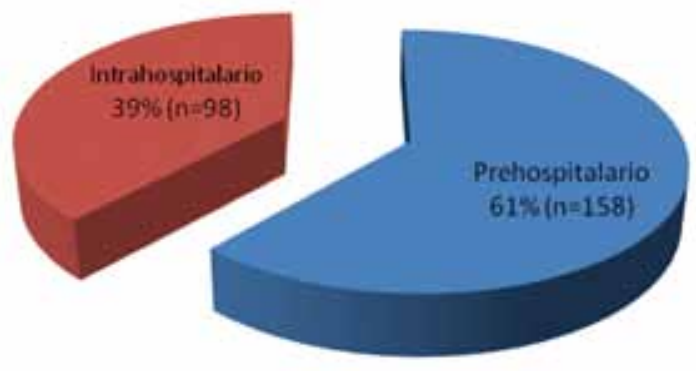

Figura 2. Momento de inicio del tratamiento analgésico: prehospitalario e intrahospitalario ( $\mathrm{y} \%$ ).

\section{Vía de analgesia}

La administración de analgesia por vía oral se empleó en 85 pacientes $(33 \%)$, por vía intravenosa en 183 pacientes $(71 \%)$, por vía intramuscular en 19 pacientes $(7 \%)$, por vía intraósea en 25 pacientes $(9 \%)$, por vía subcutánea en 7 pacientes $(2 \%)$ y por vía transmucosa en 7 pacientes (2\%). (Figura 3 ).

A 192 pacientes $(75 \%)$ se les administró analgesia por una única vía, a 50 pacientes $(19 \%)$ por dos vías, a 13 pacientes $(5 \%)$ por tres vías, a 1 paciente $(0,4 \%)$ por cuatro vías diferentes. (Figura 3 ). 


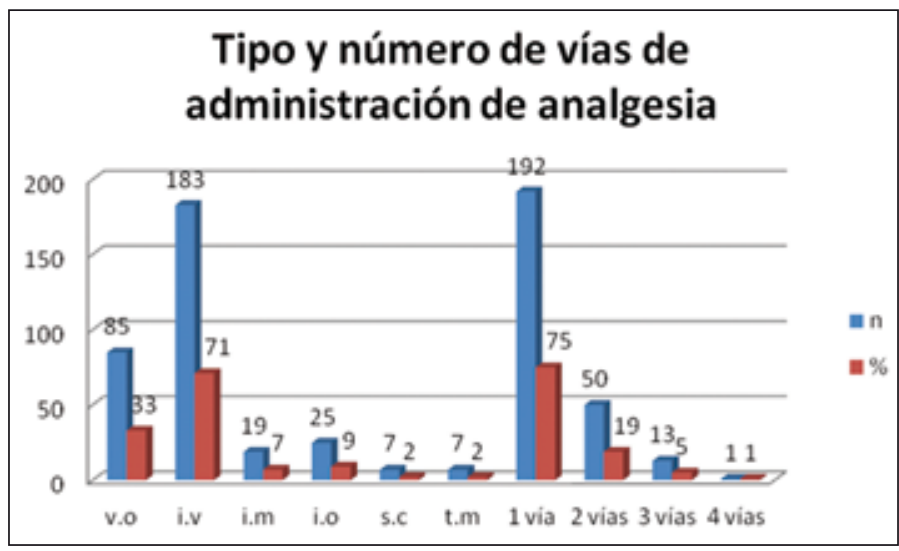

Figura 3. Tipo de vía de administración de fármacos (vía oral-v.o-, intravenosa-i.v-, intramuscular-i.m-, intraósea-i.o-, submucosa -s.c-, transmucosa -t.m-) y número de vías empleadas. (n y\%).

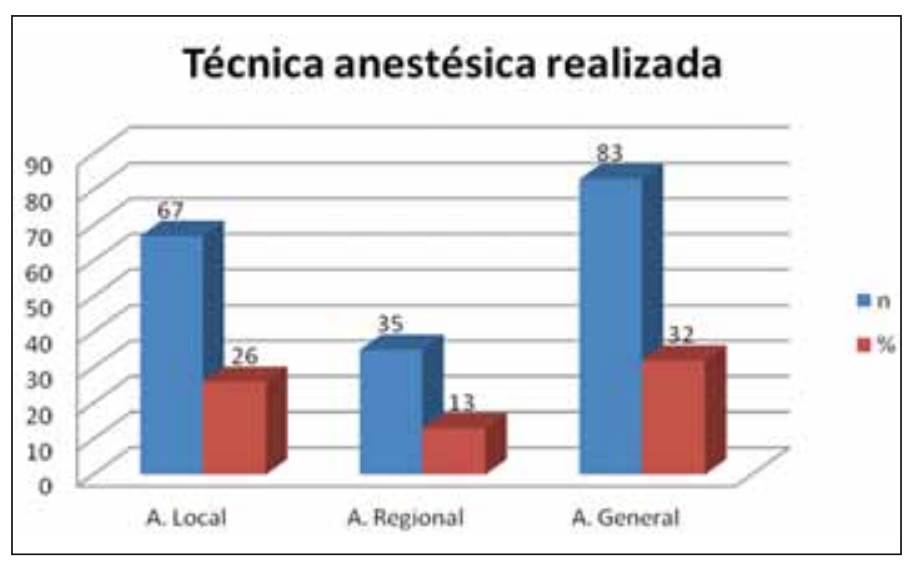

Figura 4. Técnica anestésica realizada (n y\%).

\section{Anestesia}

A 67 pacientes $(26 \%)$ se les realizó anestesia local, a 35 pacientes $(13 \%)$ anestesia regional y a 83 pacientes $(32 \%)$ anestesia general. (Figura 4).

\section{5. Índices de gravedad y analgésicos (Figuras 5 y 6 )}

Dentro del grupo de bajas catalogadas por NISS como leves $(n=174,68 \%)$, el 83\% $(n=145)$ recibieron AINEs, 19\% $(n=34)$ fueron tratados con opioides menores, $29 \%(\mathrm{n}=51)$ con opioides mayores, 16\% $(n=28)$ con coadyuvantes y $4 \%(n=8)$ con ketamina. Al 84\% ( $n=147)$ se les administró algún AINE + opioide menor, mientras que para el $91 \%(\mathrm{n}=159)$ el tratamiento elegido fue AINE+ opioide menor + opioide mayor. Al 16\% $(\mathrm{n}=28)$ se les realizó anestesia regional, mientras que al $17 \%(\mathrm{n}=31)$ se les administró anestesia general.

Dentro del grupo de bajas catalogadas por NISS como moderadas $(n=42,17 \%)$, el 33\% $(n=14)$ recibieron AINEs, $42 \%(n=18)$ fueron tratados con opioides menores, $59 \%(n=25)$ con opioides mayores, $59 \%(n=25)$ con coadyuvantes y $21 \%(n=9)$ con ketamina. Al 69\% (n=29) se les administró algún AINE + opioide menor, mientras que para el $95 \%(n=40)$ el tratamiento elegido fue AINE+ opioide menor + opioide mayor. $\mathrm{Al} \mathrm{14 \%}(\mathrm{n}=6)$ se les realizó aneste-

\section{Gravedad de los pacientes según índice NISS ( $\mathrm{n}$ y \%)}

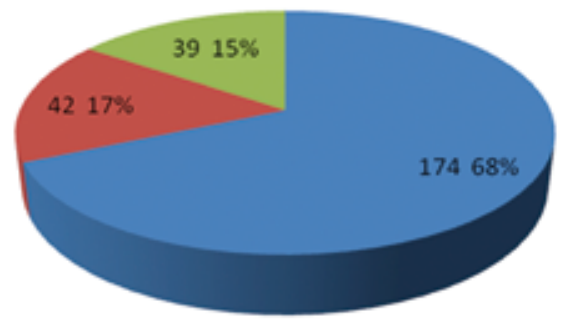

= NISSLEVE

= NISS MODERADO

E NISS GRAVE

Figura 5. Clasificación de las bajas según el índice NISS: leve, moderada y grave. (n y\%)

\section{Tratamiento analgésico administrado según gravedad}

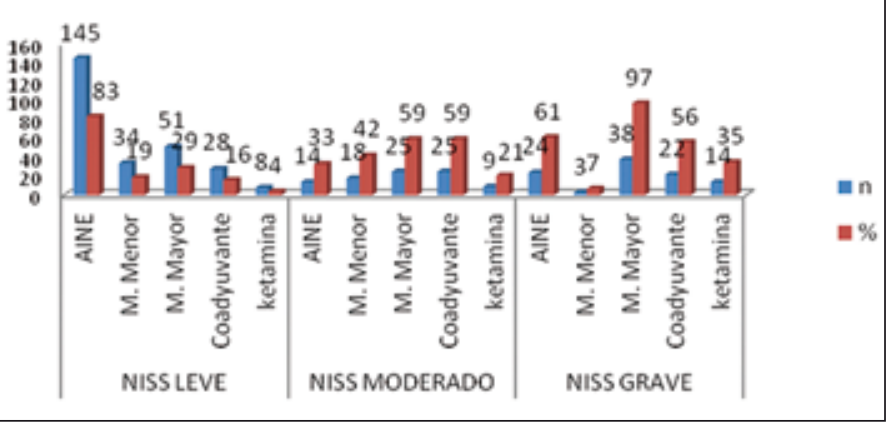

Figura 6. Tratamiento analgésico administrado según la gravedad medida por el índice NISS. ( $n$ y\%).

sia regional, mientras que al 54\% $(\mathrm{n}=23)$ se les administró anestesia general.

Dentro del grupo de bajas catalogadas por NISS como graves $(\mathrm{n}=39,15 \%)$, el $61 \%(\mathrm{n}=24)$ recibieron AINEs, $7 \%(\mathrm{n}=3)$ fueron tratados con opioides menores, $97 \%(n=38)$ con opioides mayores, $56 \%(n=22)$ con coadyuvantes y $35 \%(n=14)$ con ketamina. Al $38 \%$ $(\mathrm{n}=15)$ se les administró algún AINE + opioide menor, mientras que para el $100 \%(n=39)$ el tratamiento elegido fue AINE+ opioide menor + opioide mayor (Imagen 5 y 6$).$ Al 2\% $(n=1)$ se le realizó anestesia regional, mientras que al $74 \%(n=29)$ se les administró anestesia general.

\section{6. Índices de gravedad e inicio del tratamiento analgésico}

Dentro de las bajas catalogadas como leves, el 45\% (n=79) recibieron tratamiento pre o intrahospitalario, mientras que al $55 \%$ $(\mathrm{n}=95)$ se le administró tratamiento analgésico pre e intrahospitalario. Del grupo de bajas agrupadas por NISS como moderadas, el $7 \%(n=3)$ recibieron analgesia a nivel pre o intrahospitalario, mientras que el $92 \%(n=39)$ tuvieron tratamiento analgésico a nivel pre e intrahospitalario. Del tercer grupo según NISS (bajas graves), el $10 \%(\mathrm{n}=4)$ recibieron analgesia a nivel pre o intrahospitalario, sin embargo el $90 \%(n=35)$ recibieron analgesia a nivel pre e intrahospitalario. 


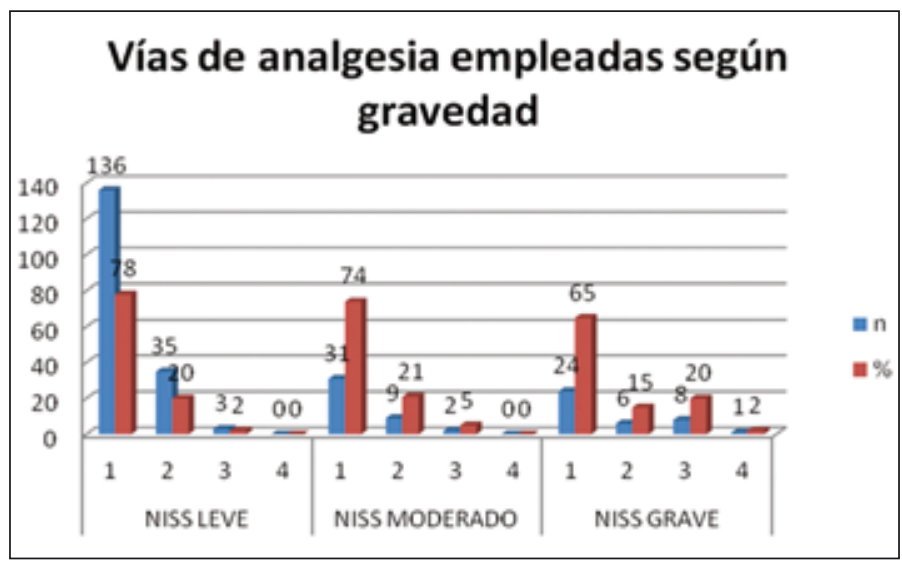

Figura 7. Vias de analgesia empleadas según el índice de gravedad NISS. (n y\%).

\section{7. Índices de gravedad y vías de administración de analgesia}

Dentro del grupo catalogado por NISS como leve, se emplearon las siguientes vías de administración de analgésicos ( 1 vía: $\mathrm{n}=136$, $78 \% ; 2$ vías: $n=35,20 \% ; 3$ vías: $n=3 ; 2 \%$; 4 vías: $n=0 ; 0 \%$. En las bajas clasificadas como moderadas ( 1 vía: $\mathrm{n}=31,74 \%$; 2 vías: $\mathrm{n}=9,21 \%$; 3 vías: $\mathrm{n}=2,5 \%$; 4 vías: $\mathrm{n}=0 ; 0 \%$ ) y en las bajas graves ( 1 vía: $\mathrm{n}=24$, $65 \%$; 2 vías: $n=6,15 \% ; 3$ vías: $n=8,20 \% ; 4$ vías: $n=1 ; 2 \%$ ) (Figura 7 ).

\section{8. Índices de gravedad y homogeneidad farmacológica}

La homogeneidad farmacológica se muestra en la tabla 3.

El grupo farmacológico de AINES, al estar compuesto por 5 fármacos distintos (paracetamol, metamizol, ibuprofeno, ketorolaco y otros) necesita un análisis particular a la hora de valorar dentro de este grupo la homogeneidad o heterogenicidad en el tratamiento (Tabla 4).

Tabla 3. Homogeneidad del tratamiento farmacológico.

\begin{tabular}{|lccc|}
\hline & \multicolumn{3}{c|}{ NISS n (\%) } \\
\cline { 2 - 4 } & Leve & Moderado & Grave \\
\hline AINES & $145(83)$ & $28(66)$ & $15(38)$ \\
AINES u Opioide menor & $147(84)$ & $29(69)$ & $15(38)$ \\
AINES u Opioide menor u Opioide mayor & $159(91)$ & $40(95)$ & $39(100)$ \\
$\begin{array}{l}\text { AINES u Opioide menor u Opioide } \\
\text { mayor o coadyuvante }\end{array}$ & $159(91)$ & $40(95)$ & $39(100)$ \\
$\begin{array}{l}\text { AINES u Opioide menor u Opioide } \\
\text { mayor o ketamina }\end{array}$ & $159(91)$ & $41(97)$ & $39(100)$ \\
\hline
\end{tabular}

Tabla 4. Homogeneidad del tratamiento farmacológico (grupo de AINES).

\begin{tabular}{|lccc|}
\hline & \multicolumn{3}{c|}{ NISS n (\%) } \\
\cline { 2 - 4 } & Leve & Moderado & Grave \\
\hline Paracetamol & $81(46)$ & $13(31)$ & $5(12)$ \\
Metamizol & $103(59)$ & $20(47)$ & $7(17)$ \\
Ibuprofeno & $27(15)$ & 0 & 0 \\
Ketorolaco & $43(24)$ & $18(42)$ & $11(28)$ \\
Otros AINES & 0 & 0 & 0 \\
\hline
\end{tabular}

\section{DISCUSIÓN}

En nuestro estudio se analiza la analgesia elegida, el momento de inicio del tratamiento, la vía de administración empleada y el tipo de anestesia realizada, tanto desde un punto de vista global, como dependiente de la gravedad de la baja valorada según el índice NISS.

De un modo global, la familia farmacológica más administrada fueron los AINEs, seguida de los mórficos mayores. Se inició la pauta analgésica a nivel prehospitalario de forma predominante, administrando a todas las bajas algún tipo de analgesia a lo largo de la cadena asistencial. En la mayoría de los casos se empleó una única vía, siendo la i.v. la preferida. La anestesia general se empleó en aproximadamente $1 / 3$ de los casos.

En cuanto a los valores obtenidos dependientes del índice NISS, destaca la tendencia a administrar fármacos de todos los grupos analgésicos a pesar de ser catalogados como casos leves, el incremento progresivo en realizar anestesia general según se aumenta la gravedad de la baja, la administración continuada pre e intrahospitalaria cuando la severidad se incrementa y la prevalencia para emplear una vía de administración de fármacos independientemente del índice obtenido.

Respecto al estudio de la homogeneidad de las variables, hemos encontrado que está presente a la hora de elegir los fármacos analgésicos de forma general y la vía de administración, sin embargo no lo es con el tipo de AINE elegido y con el momento de inicio de la analgesia.

Podemos considerar la existencia de variabilidad respeto a los AINEs debido al amplio abanico farmacológico dentro de este grupo, a la posibilidad logística real de contar con todos ellos o a la diferente experiencia de cada médico militar con cada uno de los fármacos.

En el ambiente militar, el momento de inicio de la analgesia puede estar determinado por multitud de variables como la situación táctica, el tipo de evacuación realizada, el número de bajas a atender, la distancia hasta el escalón superior, la situación clínica del paciente o la experiencia del oficial médico. Puede que uno o la suma de varios de estos factores haya sido la desencadenante de la heterogenicidad en el inicio de la analgesia de la muestra.

A pesar de seguir un patrón homogéneo, se puede considerar excesivo emplear en un $91 \%$ de las bajas catalogadas como leves: AINEs + mórficos menores + mórficos mayores. Aunque el motivo pueda ser el escaso tiempo para valorar de forma inicial a la baja, la necesidad de realizar un triaje, la búsqueda de una ansiolisis coadyuvante, la ausencia de objetivar el dolor mediante una escala analgésica o incluso la variabilidad genética y ambiental de las diferentes bajas, es necesario señalar el incremento de efectos secundarios, de costes económicos y de mantenimiento logístico que lleva aparejado el empleo sobredimensionado de fármacos.

El perfil farmacocinético y farmacodinámico de la ketamina, hacen que este fármaco pueda ser empleado frecuentemente en un ambiente hostil y austero como puede ser el militar. Sin embargo, tras el análisis de nuestra muestra, no se encuentra que su administración haya sido frecuente. Puede que el motivo de este hecho sea la falta de experiencia de los oficiales médicos con la ketamina en Territorio Nacional.

La vía intravenosa sigue siendo la más empleada para administrar la analgesia en la muestra estudiada. La utilización frecuente de este acceso puede deberse a la experiencia de los oficiales médicos o 
enfermeros y a la facilidad relativa de la técnica. Destaca del mismo modo, el empleo del acceso intraóseo en ambiente militar. Una nueva alternativa es la administración de analgesia (fentanilo) a nivel sublingual, con lo que se podrían obtener importantes ventajas en ambiente táctico.

En la mayoría de los casos, únicamente se empleó una vía de acceso de medicación. A pesar de que puede ser suficiente, siempre es adecuado valorar una segunda vía de administración con el objetivo de facilitar la administración de fármacos, reponer volumen mediante soluciones cristaloides o coloides, o por si se interrumpe el acceso durante el transporte de la baja.

La asistencia sanitaria sobre la baja en combate procedente de los actuales conflictos asimétricos, ha sufrido ciertas variaciones en cuanto al diagnóstico, tratamiento y abordaje del dolor agudo y crónico. En la actualidad, los pilares básicos del tratamiento analgésico son la instauración precoz desde el lugar del incidente y a través de todos los escalones sanitarios, incorporando terapias analgésicas multimodales (perfusión epidural o sobre nervios periféricos, agentes inflamatorios, ketamina, clonidina, mexiletina, anticonvulsionantes, antidepresivos, ansiolíticos...) $)^{12}$. Según la revisión realizada por Malchow ${ }^{13}$, una estrategia más agresiva sobre el dolor agudo, ha provocado un descenso del dolor agudo y crónico de las bajas en ambiente militar.

Estos datos coinciden con el estudio de Ling ${ }^{14}$ sobre los cambios sanitarios realizados durante la operación Iraqi Freedom (Irak) y Enduring Freedom (Afganistán). Entre ellos, la mejora del tratamiento del dolor ha provocado que la supervivencia sea muy superior a anteriores conflictos ocurridos durante el siglo XX.

Looker ${ }^{15}$ aconseja el empleo de un índice de graduación del dolor diseñado en el ROLE 4 británico de Birmingham (Tabla 5). Sus ventajas respecto a otros índices más complejos como la Escala Visual Analógica, McGill, EQ5D, SF-36 son la facilidad de uso, la posibilidad de empleo a lo largo de la evacuación y la no necesidad de emplear signos vitales (frecuencia cardiaca, frecuencia respiratoria, temperatura...) para cumplimentar los datos del índice.

Mercer $^{16}$ evaluó el dolor, el tratamiento analgésico empleado y el grado de satisfacción de 50 bajas británicas procedentes de Irak y Afganistán desde mayo a noviembre de 2007. Mediante la escala visual analgésica, los pacientes valoraron el dolor a nivel prehospitalario, hospitalario, durante la evacuación a Territorio Nacional y por último en el ROLE 4 en Birmingham (Reino Unido). Para el diagnóstico de dolor neuropático, se empleó la escala LANSS (Leeds Assessment of Neuropathic Symptoms and Signs). La analgesia administrada fue: paracetamol (100\%), morfina (86\%), diclofenaco $(72 \%)$, codeína $(56 \%)$, oxicodona $(48 \%)$, tramadol (42\%), pregabalina (32\%), amitriptilina (30\%), ibuprofeno (12\%) y gabapentina (10\%). El 30\% de las bajas presentaron signos y síntomas sugestivos de dolor neuropático. En el $4 \%$ de las bajas, se empleó anestesia regional (anestesia epidural o bloqueo nervioso a nivel femoral).

Tabla 5. Índice de graduación del dolor diseñado en el ROLE 4 británico de Birmingham.

\begin{tabular}{|cll|}
\hline Valor & \multicolumn{1}{c|}{ Nivel del dolor } & \multicolumn{1}{c|}{ Analgesia } \\
\hline 3 & Dolor continuo en reposo, grave en movimiento & Morfina y anteriores \\
2 & Dolor en reposo, moderado en movimiento & Opioides y AINEs \\
1 & No dolor en reposo, moderado en movimiento & Paracetamol \\
0 & No dolor, tanto en reposo como en movimiento & Ninguna \\
\hline
\end{tabular}

Un estudio analizó mediante una encuesta electrónica, la analgesia administrada por anestesiólogos, médicos de urgencia, enfermeras y paramédicos británicos desplegados en Irak y Afganistán en 2006. Se obtuvieron 122 cuestionarios. El 52\% opinó que la morfina i.m no era el analgésico ideal, debido a su prolongado inicio de acción. En cuanto a la principal característica que debía tener el analgésico ideal para el combate, el $83 \%$ de los encuestados señaló la facilidad de empleo y fiabilidad del fármaco. La vía de administración predilecta fue la vía intranasal (72\%). El 91\% de los interrogados, admitieron que no contaban con experiencia suficiente para evaluar los anestésicos volátiles ${ }^{17}$.

Stojadinovic ${ }^{18}$ describe el empleo de 646 procedimientos de anestesia regional sobre bajas en combate procedentes de Irak. En 126 heridos, a los que se les había realizado un bloqueo nervioso periférico continuo, se constató un descenso significativo del dolor a los 7 días de instauración del tratamiento $(\mathrm{p}<0,001)$, en el $11 \%$ de las bajas aparecieron complicaciones menores relacionadas con la colocación y manipulación del catéter, y la tasa de infección fue del $1,9 \%$.

En un artículo de Clark ${ }^{19}$ publicado en 2009, se analizan 129 bajas procedentes de Irak y Afganistán, que habían sufrido politraumas graves. Los heridos se dividieron según el agente lesional y el tipo de tratamiento analgésico (intesivo, interdisciplinar y tratamiento en unidad del dolor). El resultado obtenido fue que las bajas por blast necesitaron más analgesia, una estancia hospitalaria más prolongada, una mejoría menor tras el tratamiento analgésico administrado y una tasa de trastornos postraumáticos mayor que en otros grupos.

El estudio de Buckenmaier ${ }^{20}$ analiza el empleo de bloqueo continuo de nervios periféricos durante la evacuación y estancia en el ROLE 4 de Landstuhl (Alemania), de 110 militares norteamericanos heridos procedentes de Irak y Afganistán entre julio de 2007 y febrero de 2008 . En el $60 \%$ de los casos, el agente lesional fue el artefacto explosivo improvisado. El incremento del dolor se asoció con un aumento de la ansiedad y con un empeoramiento clínico durante el transporte. El empleo de catéteres de bloqueo continuo en nervios periféricos, proporcionaron una mejora en la sensación álgica $(\mathrm{p}=0,02)$.

La experiencia británica sobre el empleo de bloqueo continuo sobre nervios periféricos queda patente en el trabajo de Hughes ${ }^{21}$. Este autor describe el empleo de 51 bloqueos realizados en el ROLE-2E británico de Camp Bastion (Afganistán). En 26 de ellos, se empleó un catéter. La mayoría de éstos requirieron algún tipo de AINE como analgésicos de rescate, pero a ninguno se le administró un opioide, se disminuyeron por tanto los efectos no deseables de los mórficos.

El anestesiólogo en ambiente militar, necesita habituarse a la administración de diferentes tipos de analgesia empleando distintas técnicas y fármacos. Esto no quiere decir que el cuidado analgésico a la baja de combate sea reducido. El médico militar debe estar en búsqueda constante de nuevas tecnologías, nuevas técnicas y nuevas evidencias que aseguren el mejor cuidado posible a la baja en combate ${ }^{22}$.

Algunas de las lecciones aprendidas procedentes del tratamiento de las bajas en combate, enseñan la necesidad de un correcto y moderno tratamiento analgésico. Para ello es necesaria la adecuada preparación, formación y entrenamiento de los anestesiólogos incluidos en equipos multidisciplinares que trabajen en ROLE $4^{23}$. El aprendizaje y práctica de protocolos médicos en Territorio Nacional 
son fundamentales a la hora de normalizar el empleo de dispositivos y medicación en zona de operaciones ${ }^{24}$.

Como primera medida analgésica, algunos autores prefieren los sistemas de inyección rápida de morfina vía intramuscular y fentanilo transmucoso, administrados por parte de personal entrenado no médico. Posteriormente, recomiendan el empleo de la vía intravenosa para emplear opioides mayores, AINEs, ketamina, así como técnicas de anestesia regional (vgr; bloqueo del neuroeje, plexo y nervio periférico $)^{25}$. Sin embargo, en otros estudios se insiste en la administración i.v.de morfina en las bajas de combate en las que es necesaria la analgesia ${ }^{26}$.

El tratamiento analgésico administrado por paramédicos norteamericanos sobre 22 heridos pertenecientes a diferentes fuerzas de operaciones especiales desplegados en Irak durante el año 2003, fue descrito por Kotwal ${ }^{27}$. En su estudio, el fentanilo transmucoso aparece como alternativa analgésica para pacientes aislados, aconsejando la vía intravenosa para aquellos heridos inestables hemodinámicamente.

La mejora en la formación en el acceso i.v y en el uso de los nuevos dispositivos intraóseos ha mejorado igualmente la administración y el aporte de analgesia ${ }^{28,29}$.

Generalmente, el profesional sanitario militar norteamericano está entrenado para administrar morfina por vía intramuscular e intravenosa. Además se les ha enseñado la administración de citrato de fentanilo por vía transmucosa oral ${ }^{30}$.

La educación y el entrenamiento para la realización de bloqueos nerviosos periféricos también se están empezando a aplicar en el entorno prehospitalario de combate por parte de los profesionales asistenciales de las fuerzas especiales norteamericanas, especialmente en lo que se refiere a las lesiones de las extremidades ${ }^{31}$.

La infiltración local de la herida y los bloqueos nerviosos básicos como los de fascia iliaca, intercostal o supraclavicular realizados antes del traslado de la baja, pueden proporcionar una analgesia profunda. Estos bloqueos también se acompañan de un cociente riesgo-beneficio muy bajo. La aplicación de la anestesia regional, es una técnica importante en el tratamiento de las bajas en combate. Cuando se lleva a cabo en el contexto prehospitalario del campo de batalla, la anestesia regional no causa alteraciones del estado respiratorio o mental, y permite que el soldado pueda realizar algunas tareas mínimas mientras espera su evacuación ${ }^{32}$.

En un estudio sobre 500 bajas recogidas desde marzo del 2003 hasta diciembre 2004, que fueron evacuadas al ROLE 4 (Walter Reed Army Medical Center), con lesiones en miembros provocadas por artefactos explosivos, se describe que 287 (57\%) recibieron anestesia regional, empleándose el bloqueo de nervio periférico (en bolus y continuo) y bloqueo epidural con y sin catéter ${ }^{33}$.

Además de los bloqueos nerviosos periféricos de inyección única, también se pueden llevar a cabo técnicas regionales avanzadas en miembros y sobre el neuroeje. En un estudio en el que se evaluó y trató el dolor en 38 soldados combatiente en Irak utilizando para ello técnicas más avanzadas (esteroides epidurales, bloqueos de las carillas articulares y la farmacoterapia dirigida por un médico especializado en dolor), la vuelta a las tareas de combate tuvo lugar en aproximadamente el $80 \%$ de los casos. Esta cifra difiere de la tasa de reanudación observada en soldados a los que se trata de modo convencional en el escenario del incidente $(50 \%)$ y se diferencia notablemente de los militares tratados en un centro asistencial ubicado en Estados Unidos, ya que son escasos los efectivos de este último grupo que vuelven al combate ${ }^{34}$.
Estas técnicas regionales en ambiente militar, pueden verse implementadas notablemente gracias al empleo en Zona de Operaciones de ecografía y radioscopia que facilitan el control de los bloqueos nerviosos avanzados ${ }^{35}$.

En muchas de las instalaciones sanitarias norteamericanas desplegadas en el Teatro de Operaciones, se cuenta con dispositivos portátiles de analgesia controlada por el paciente PCA (patient-controlled analgesia) que pueden ser transportados por aire y que están alimentados por baterías. Estas bombas de PCA, pueden acompañar al paciente en todo el espectro asistencial ${ }^{9,36}$.

Los dispositivos como la bomba de PCA cutánea con fentanilo, pueden no solo facilitar una analgesia eficaz, sino también reducir algunas de las carencias terapéuticas (ansiolisis, sedación...) que habitualmente están amplificadas en los ambientes austeros como es el militar ${ }^{37}$

Las modalidades principales de analgesia empleadas en los vuelos de evacuación a Territorio Nacional son los bolos intermitentes de morfina, los dispositivos PCA y los catéteres para la administración continuada ${ }^{12}$.

La analgesia por vía oral también es una opción. Los medicamentos orales en combinación muestran sinergismo, proporcionan una analgesia multimodal, facilitan la disminución de las dosis de opiáceos y no impiden que el soldado pueda llevar su arma9. La mayor parte de las fuerzas de operaciones especiales que actúan en ambientes prehospitalarios de combate, llevan encima una «bosa de píldoras». Esta bolsa contiene meloxicam y paracetamol, para que el propio soldado que sufre una lesión dolorosa se abstenga ${ }^{38}$.

En la actualidad, los sanitarios de las fuerzas de operaciones especiales cuentan con fentanilo transmucoso. Se utiliza una dosis inicial de $400 \mu \mathrm{g}$, que generalmente da lugar a concentraciones plasmáticas máximas no superiores a $2 \mu \mathrm{g} / \mathrm{ml}$. Esta concentración plasmática se asocia a un incremento del riesgo de depresión respiratoria. Dado que alcanza sus concentraciones séricas máximas aproximadamente a los 30 minutos, la nueva dosis se puede iniciar 15 minutos después de que ha finalizado la administración de la dosis previa ${ }^{39}$. Otro autor ha demostrado que el citrato de fentanilo oral de administración transmucosa alivia el dolor de intensidad moderada en el campo de batalla ${ }^{27}$. A partir de 2009, el fentanilo también se puede administrar por vía sublingual y nasal, pudiendo considerarse en el futuro como otra nueva vía alternativa de administración de la analgesia ${ }^{40}$.

La ketamina también se ha utilizado con buenos resultados como analgésico prehospitalario en el contexto de combate ${ }^{41}$. Administrada con dosis subanestésicas, este fármaco consigue un intenso efecto de alivio del dolor, una potenciación del efecto de los opiáceos, una prevención de la hiperalgesia y cuenta con un importante margen de seguridad ${ }^{42-45}$.

Las recomendaciones iniciales en el Tactical Casuality Combat Care sobre la analgesia en combate, incluían la morfina i.v o i.m. En 2003, se propusieron los AINEs, pero éstos fueron sustituidos por los efectos deletéreos que podían provocar sobre la coagulación. Por este hecho, a partir de las guías de 2006 se introduce el empleo de paracetamol y meloxicam. Otra novedad de ese año fue la inclusión del fentanilo transmucoso como una importante alternativa en el tratamiento analgésico de la baja en combate ${ }^{46}$.

En los últimos años el Ministerio de Defensa español ha apostado por compromisos dentro del seno de la comunidad internacional. Por ello, el curso del despliegue en el Teatro de Operaciones y las 
necesidades del combatiente del futuro obligan a la Medicina Militar a adaptarse a los nuevos retos, problemas y paradigmas que demanda el combatiente del futuro ${ }^{47}$.

En la actualidad, la Sanidad Militar española cuenta con la posibilidad de desplegar 4 escalones sanitarios o ROLEs. Siendo ROLE 1, la instalación sanitaria más sencilla y ROLE 4 la más compleja. Esta última se localiza normalmente en Territorio Nacional ${ }^{48,49}$.

El primer análisis español reciente sobre el papel del anestesiólogo-reanimador lo realiza Muñoz-Mingarro ${ }^{50}$. Destaca que deben definirse tres niveles de reanimación, con una estricta y rigurosa protocolización de las actuaciones sanitarias, que permita correlacionar los niveles de reanimación con la cualificación del personal encargado de actuar. Señala que los oficiales médicos anestesiólogos deben estar en continua actualización, con una formación permanente, que permita renovar las normas y planes de enseñanza en función de la experiencia acumulada y la aparición de nuevos datos, fármacos y protocolos de actuación que vayan apareciendo.

En un trabajo posterior, se describe las cantidades estimadas para una formación quirúrgica móvil, con la intención de sistematizar las necesidades de material y fármacos desde un punto de vista anestesiológico para el tratamiento pre, intra, postoperatorio y de la terapia del dolor, de forma que posibilite la asistencia de aproximadamente cien bajas, antes de que sea necesario la reposición de material ${ }^{7}$.

En 1995, el Estado Mayor de la Defensa ${ }^{11}$, define la analgesia y sedación en cada uno de los escalones sanitarios, tal y como se muestra la Tabla 6.

Este protocolo se mantiene vigente hasta el estudio de Muñoz ${ }^{51}$, que define el tipo de sedoanalgesia y relajación muscular durante la puesta en estado de evacuación y traslado.

El tratamiento del dolor y la aplicación de técnicas de anestesia, actualmente, en Fuerzas Armadas españolas se sustenta en la prescripción médica desde el ROLE I al IV, basándose en el escalón terapéutico de la OMS. Reserva las técnicas de anestesia regional para personal médico anestesiólogo en ROLE II o superiores. Sin embargo no se ha encontrado ningún caso publicado por parte de anestesiología militar española al respecto.

El material analgésico propuesto para el tratamiento de una baja durante la aeroevacuación, queda definido en un trabajo publicado en 2006. Se considera necesario que en la aeronave se disponga de toradol, cloruro mórfico $2 \%$, fentanilo y ketamina en ampollas ${ }^{52}$

Tabla 6. Analgesia de cada escalón sanitario.

\begin{tabular}{|ll|}
\hline Escalón & Analgesia \\
\hline $\begin{array}{l}\text { Primer escalón } \\
\text { Primer escalón } \\
\text { (puesto de auxilio de Batallón) }\end{array}$ & Dolor ligero: AAS/Paracetamol \\
& Dolor fuerte: Morfina $20 \mathrm{mg}$ IM \\
& Diazepam. \\
Segundo escalón & Oral: Sulfato de morfina (comprimidos) \\
& Parenteral: Sulfato de morfina 2,5 mg. \\
& Sedación oral: Diazepam 5 mg \\
& Sedación parenteral: Diazepam/Midazolam \\
& Ketamina (+Atropina+ Midazolam) \\
& Ketamina \\
Tercer escalón & Considerar opioides epidurales y \\
& anestésicos locales epidurales con catéter. \\
&
\end{tabular}

Este trabajo presenta las siguientes limitaciones. En primer lugar es un estudio retrospectivo realizado en Zona de Operaciones, por lo que cuenta con condicionantes tácticos y logísticos que han podido interferir en la toma de decisiones médicas sobre las bajas.

En segundo lugar, su diseño carece de una escala de valoración del dolor inicial que pudiera estratificar de algún modo, la cantidad de dolor que padece la baja a lo largo del proceso asistencial.

\section{CONCLUSIONES}

El tratamiento analgésico prestado a las bajas en combate de la muestra es homogéneo en cuanto a los fármacos empleados, el tipo y número de vías de administración conseguidas. Sin embargo es heterogéneo en cuanto al tipo de AINE elegido y al inicio en la administración de los fármacos analgésicos.

\section{AGRADECIMIENTOS}

A Carlos Gutiérrez Ortega por su apoyo incondicional a la hora de realizar este trabajo. A los compañeros desplegados en Afganistán.

\section{BIBLIOGRAFÍA}

1. Houghton IT. Some observations on early military anaesthesia. Anaesth Intensive Care 2006; 34: 6-15.

2. Armero P, Carrillo F. Afganistán. En: El Ejército del Aire en Operaciones de Paz. Madrid: Ministerio de Defensa; 2004. p. 61-76.

3. Navarro R, Bartolomé E, Jara I, Oreja A, González G. Capacidades y asistencia sanitaria realizada por el ROLE-2 español en la FSB de Herat (Afganistán) desde febrero a julio del 2007. Sanid. Mil. 2008; 64 (2): 98-104.

4. Astudillo W, Mendinueta C, Astudillo E, Gabilondo S. Principios básicos para el control del dolor total. Rev Soc Esp Dolor 1998; 6: 29-40.

5. Mabrey RL. Battlefield Medications. En: Bond C, editor. 68W Advanced Field Craft Combat Medic Skill. Massachusetts: Jones and Barlett Publishers; 2009. p. 190-219.

6. Connor DJ, Ralph JK, Aldington DJ. Field Hospital Analgesia. JR Army Med Corps 2009; 155(1): 49-56.

7. Muñoz-Mingarro J, Ortiz J, Sierra F. Anestesia en campaña. Material y fármacos. Propuesta para una formación quirúrgica móvil (FQM). Med Mil 1993; 49: 100-13.

8. Zarazaga A, García de Lorenzo A. Escalas e índices pronósticos en el politraumatizado y quemado crítico. En: Rodríguez J. El politraumatizado. Diagnóstico y terapéutica. 1. ${ }^{a}$ ed. Madrid: Ed. Universitaria Ramón Areces; 2008. p. 37-54

9. Black IH, McManus J. Tratamiento del dolor en las operaciones de combate actuales. Prehospital Emergency Care 2009; 3: 223-7.

10. Flutter C, Ruth M, Aldington D. Pain management during Royal Air Force Strategic Aeromedical Evacuations. JR Army Med Corps 2009; 155(1): 42-6.

11. EUROMED. Cuidados de urgencia en campaña, principios y protocolo. Ministerio de Defensa; 1995.

12. Buckenmaier CC, McKnight GM. Continuous peripheral nerve block for battlefield anesthesia and evacuation. Reg Anesth Pain Med 2005. 30: 202-5.

13. Malchow RJ, Black IH. The evolution of pain management in the critically ill trauma patient: Emerging concepts from the global war on terrorism. Crit Care Med 2008; 36: 346-54.

14. Ling GS. Rhee P. Ecklund JM. Surgical innovations arising from the Iraq and Afghanistan wars. Annu Rev Med 2010; 61: 457-68.

15. Looker J, Aldington D. Pain Scores, as easy as counting to three. JR Army Med Corps 2009; 155: 42-3.

16. Mercer SJ, Chavan S, Tong JL, Connor DJ, de Mello WF. The early detection and management of neuropathic pain following combat injury. J R Army Med Corps 2009; 155: 94-8. 
17. Smith JE, Russell R, Mahoney PF, Hodgetts TJ. What is the ideal pre-hospital analgesic? A questionnaire study. JR Army Med Corps 2009; 155: 44-6.

18. Stojadinovic A, Auton A, Peoples GE, McKnight GM, Shields C, Croll SM, etal Responding to challenges in modern combat casualty care: innovative use of advanced regional anesthesia. Pain Med 2006; 7: 330-8.

19. Clark ME, Walker RL, Gironda RJ, Scholten JD. Comparison of pain and emotional symptoms in soldiers with polytrauma: unique aspects of blast exposure. Pain Med. 2009;10: 447-55.

20. Buckenmaier CC, Rupprecht C, McKnight G, McMillan B, White RL, Gallagher RM. Pain following battlefield injury and evacuation: a survey of 110 casualties from the wars in Iraq and Afghanistan. Pain Med 2009;10: 1487-96.

21. Hughes S, Birt D. Continuous peripheral nerve blockade on op herrick 9. JR Army Med Corps 2009; 155: 57-8.

22. Mahoney PF, McFarland CC. Field anesthesia and military Injury. En: Smith CE Trauma anesthesia. New York: Cambridge University Press; 2008: 343-59.

23. Edwards D, Bowden M, Aldington DJ. Pain management at ROLE 4. JR Army Med Corps 2009; 155: 58-63.

24. Mercer SJ. Whittle CL, Mahoney PF. Lessons from the battlefield: human factors in defence anaesthesia. BJA 2010; 105: 9-20.

25. Hocking G, De Mello, WF. Battlefield analgesia, a basic approach. J R Army Med Corps. 1996; 142(3): 101-2.

26. Butler FJ, Hagmann JH, Richard DT. Tactical management of urban warfare casualties in special operations. Mil Med 2000; 165: 1-48.

27. Kotwal RS, O'Connor KC, Johnson TR, Mosely DS, Meyer DE, Holcomb JB. A novel pain management strategy for combat casualty care. Ann Emerg Med 2004; $4: 121-7$

28. Timboe HL, Bruttig SP, Ruemmler MW. Adult IO in the combat zone: the past, present and future use of intraosseous infusion by the U.S. military. JEMS 2005 30: $27-8$.

29. Calkins MD, Fitzgerald G, Bentley TB, Burris D. Intraosseous infusion devices: a comparison for potential use in special operations. J Trauma 2000; 48: 1068-74.

30. Black IH, McManus J. Tratamiento del dolor en las operaciones de combate actuales. Prehospital Emergency Care 2009; 3: 223-7.

31. Calkins MD, Kuzma PJ, Larkin TM, Green DL. Pain management in the specia operations environment: regional anesthetics. Mil Med 2001 Mar; 166(3): 211-6.

32. Clinkscales CP. Anesthesia in the military settings. En: Urman RD, Gross WL, Philip BK. Anesthesia outside of the operating room. New York: Oxford University Press; 2011. p. 267-79.

33. Hoge C, McGurk D, Thomas JL, Cox AL, Engel CC, Castro CA. Mild traumatic brain injury in U.S. soldiers returning from Iraq. N Engl J Med. 2008: 31; 358(5): 453-63.

34. Clark ME, Scholten JD, Walker RL, Gironda RJ. Assessment and treatment of pain associated with combat-related polytrauma. Pain Med 2009; 10(3): 456-69.

35. Malchow RJ. Ultrasonography for advanced regional anesthesia and acute pain management in a combat environment. US Army Med Dep 2009; 5: 64-6.
36. Cohen SP, Griffith S, Larkin TM, Villena F, Larkin R. Presentation, diagnoses, mechanisms of injury, and treatment of soldiers injured in Operation Iraqi Freedom: an epidemiological study conducted at two military pain management centers. Anesth Analg 2005;101: 1098-103.

37. Carr DB, Reines HD, Schaffer J, Polomano RC, Lande S. The impact of technology on the analgesic gap and quality of acute pain management. Reg Anesth Pain Med 2005; 30: 286-91.

38. Buttar NS, Wang KK. The «aspirin» of the new millennium: cyclooxigenase-2 inhibitors. Mayo Clin Proc 2000; 75: 1027-38.

39. Egan TD, Sharma A, Ashburn MA, Kievit J, Pace NL, Streisand JB. Multiple dose pharmacokinetics of oral transmucosal fentanyl citrate in healthy volunteers. Anesthesiology 2000; 92: 665-73.

40. Davis MP. Recent development in therapeutics for breakthrough pain. Expert Rev Neurother 2010; 10: 757-73.

41. Wedmore IS, Johnson T, Czarnik J, Hendrix S. Pain management in the wilderness and operational setting. Emerg Med Clin North Am. 2005 May; 23(2): 585-601.

42. Himmelseher S, Durieux ME. Ketamine for perioperative pain management Anesthesiology 2005; 102: 211-20.

43. Subramaniam K, Subramaniam B, Steinbrook RA. Ketamine as adjuvant analgesic to opioids: a quantitative and quialitative systematic review. Anesth Analg 2004; 99: 482-95.

44. Nadeson R, Tucker A, Bajunaki E, Goodchild CS. Potentiation by ketamine of fentanyl antinociception. An experimental study in rats showing tant ketamine administered by non-spinal routes targets spinal cord antinociceptive system. $\mathrm{Br}$ J Anaesth 2002; 88: 685-91.

45. Koppert W, Sittl R, Scheuber K, Alsheimer M, Schmelz M, Schuttler J. Differential modulation of remifentanil-induced analgesia and postinfusion hyperalgesia by S-ketamine and clonidine in humans. Anesthesiology 2003; 99: 152-9.

46. Butler FK, Holcomb JB, Giebner SD, McSwain NE, Bagian J. TCCC: Evolving concepts and battlefield experiences. Mil Med 2007; 172: 1-19.

47. Sirvent G. Terrorismo y delincuencia transnacional organizada en el siglo XXI. En: Nuevos riesgos para la sociedad del futuro. Madrid: Cuadernos de Estrategia; 2003. p. 36-43.

48. Campillo JR. Bases históricas del escalonamiento del Servicio de Sanidad en operaciones. Sanid Mil 2008; 64 (1): 43-51.

49. Ejército de Tierra español. Sanidad en operaciones PD4-616. Madrid: Mando de Adiestramiento y Doctrina; 2011.

50. Muñoz-Mingarro J, Ortiz J. Reanimación en campaña: un análisis (el anestesiólogo-reanimador en el frente). Med Mil 1992; 48: 380-90.

51. Muñoz A, Maimir F. Primera asistencia a la baja en combate: Analgesia en campaña. Sanid mil 2008: 64: 21-2.

52. Maimir F, Hernández A. Metodología y material en el transporte sanitario militar en área de operaciones. Med Mil 2006; 62: 32-8. 